\title{
Congenital Adrenal Hypoplasia and Isolated Gonadotropin Deficiency
}

\author{
Gad B. Kletter, Jerome L. Gorski, and Robert P. Kelch
}

Congenital adrenal hypoplasia with gonadotropin deficiency is a rare $X$-linked recessive disorder that usually manifests with symptoms of adrenal insufficiency early in infancy. Adequate replacement therapy with glucocorticoids, mineralocorticoids, and salt has resulted in an increased survival. Slow growth and failure to undergo sexual maturation during the adolescent years usually ensues, secondary to hypogonadotropic hypogonadism. The X-linked congenital adrenal hypoplasia locus has been mapped to region Xp21.3-p21.2. Interstitial deletions of the $X$ chromosome overlapping this region have been observed to cause complex clinical problems, with adrenal hypoplasia as a prominent component. Within a family segregating the disease, there is a 50\% risk of having an affected male and a 50\% risk of having a carrier female; considerations of genetic heterogeneity, possible chromosomal abnormalities, and prenatal diagnostic studies warrant medical genetic evaluations. The following case presentations illustrate the clinical spectrum of this condition. (Trends Endocrinol Metab 1991; 2:123-128)

\section{- Case Presentation 1}

A 6-week-old infant was admitted to a local hospital because of weight loss and severe failure to thrive. Gestation and delivery were normal; birth weight was 4600 g. After receiving intravenous (i.v.) hydration he recovered and did well until -3 years of age, when he began to require frequent hospitalizations for recurrent vomiting, hyperkalemic, and hyponatremic dehydration, lethargy, and weakness. The diagnosis of primary adrenal insufficiency was made and replacement therapy with hydrocortisone succinate and 9- $\alpha$-fluorohydrocortisone was begun. Subsequently, he was hospitalized sev-

Gad B. Kletter, Jerome L. Gorski, and Robert $P$. Kelch are at the Department of Pediatrics, University of Michigan, Ann Arbor, MI 48109 0718 , USA. eral times because of seizures and unresponsiveness associated with hypoglycemia and hyponatremia. Two brothers had died suddenly at the ages of 13 months and 13 days, respectively. A maternal grandmother had two brothers who died in early childhood, and a sister of that grandmother had two sons who died in early childhood from "hyponatremic dehydration."

At age 16, when he initially came to our institution, he was a thin, short boy with a eunuchoid body habitus. Physical examination revealed a scant amount of pubic hair and minimal scrotal thinning. Pituitary gonadotropin responses to synthetic GnRH $(2.5 \mu \mathrm{g} / \mathrm{kg})$ were low [mean basal luteinizing hormone (LH) $3.9 \mathrm{IU} / \mathrm{L}$, maximal increment $2.9 \mathrm{IU} / \mathrm{L}$; and mean basal follicle-stimulating hormone (FSH) $1.9 \mathrm{IU} / \mathrm{L}$, maximal increment $1.6 \mathrm{IU} / \mathrm{L}]$. Serum testosterone was $2.1 \mathrm{nmol} / \mathrm{L}$, and bone age was 13 years.
He was admitted to the Clinical Research Center for detailed evaluation of gonadotropin secretion and responses to pulsatile administration of synthetic GnRH $(0.025 \mu \mathrm{g} / \mathrm{kg}$ per dose every $2 \mathrm{~h}$ for 5 days). His response to synthetic GnRH was minimal (Figure 1); serum LH $\alpha$ subunit did not increase (data not shown). He was started on therapy with depot testosterone enanthate, and full masculinization ensued.

\section{- Case Presentation 2}

A 7-year-old boy was referred because of vomiting and increasing fatigue over the past 3 months. He had not experienced weight loss or diarrhea. Family history was noncontributory. On admission, his weight was $30.2 \mathrm{~kg}$ (90th percentile for age) and his height was $130 \mathrm{~cm}(80 \mathrm{th}$ percentile). Physical examination revealed increased skin pigmentation, especially of the elbows, knees, knuckles, and gingivae. Serum $\mathrm{Na}^{+}$was $121 \mathrm{meq} / \mathrm{L}$ and serum $\mathrm{K}^{+}$was 5.2 meq/L. Serum cortisol was $193 \mathrm{nmol} / \mathrm{L}$ (normal, 28-662 nmol/ L) and did not increase after stimulation with adrenocorticotrophic hormone (ACTH). Urinary 17-hydroxysteroid excretion was low. Plasma ACTH was 6300 $\mathrm{ng} / \mathrm{L}$ (normal, <200 ng/L). Primary adrenal insufficiency was diagnosed, and therapy with mineralo- and glucocorticoids was initiated. Evaluation of a possible autoimmune endocrinopathy included determination of all of the following antibody titers, all of which were undetectable: antiadrenal, antiislet cell, antimitochondrial, anti-smooth-muscle, antiparietal cell, antithyroglobulin, and antimicrosomal. Seven years later, failure to develop secondary sex characteristics prompted further evaluation. At $15^{1 / 2}$ years of age, his external genitalia showed no pubertal changes: there was a scant amount of pubic hair, and the phallus and testes were prepubertal in size. Bone age was 13 years; serum gonadotropin concentrations were somewhat low (FSH 4.2 and LH $1.6 \mathrm{KU} / \mathrm{L}$ ); serum testosterone was undetectable. The diagnosis of hypogonadotropic hypogonadism was suspected, and he was admitted for evaluation of gonadotropin 


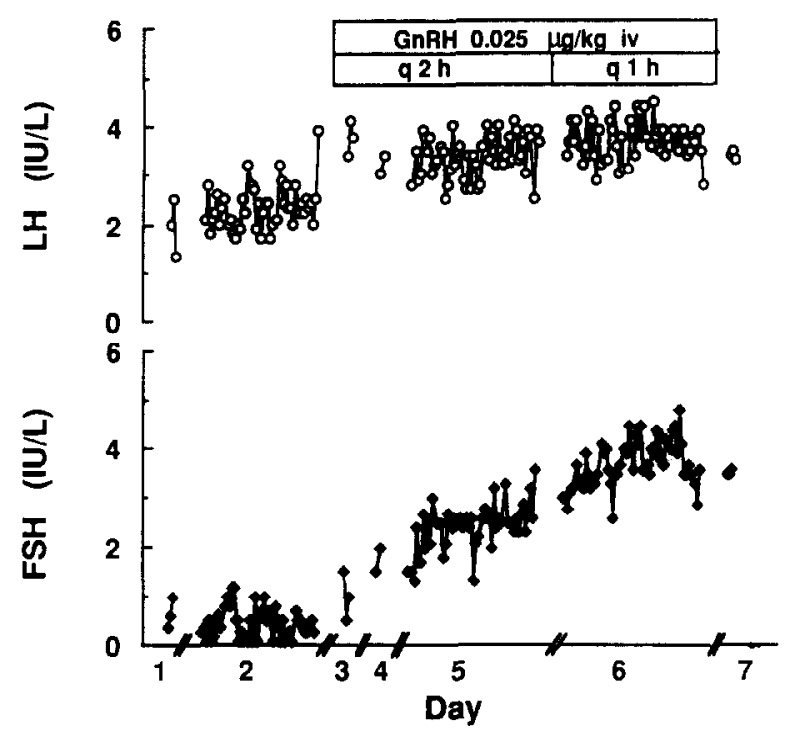

Figure 1. Plasma immunoreactive gonadotropin patterns in response to pulsatile administration of synthetic GnRH in case 1. Gonadotropin values are expressed as $I U / L$ of the second IRP-hMG standard.

secretion before and after 5 days of exogenous $\mathrm{GnRH}$ given intravenously every $2 \mathrm{~h}(0.025 \mu \mathrm{g} / \mathrm{kg}$ per dose $)$. The results are depicted in Figure 2. Pulsatile administration of synthetic GnRH resulted in an increase in FSH secretion, but no change in LH secretion. He then received human chorionic gonadotropin (hCG), $2000 \mathrm{U}$ intramuscularly (i.m.) every 3 days for 8 weeks, which resulted

in an increase of serum testosterone from undetectable to $2.1 \mathrm{nmol} / \mathrm{L}$, a poor response. Subsequently he received therapy with gradually increasing doses of the long-acting testosterone ester, testosterone enanthate (up to $200 \mathrm{mg}$ i.m. every 4 weeks), with induction of masculinization. At 20 years of age, his physical examination revealed Tanner stage-3 penile development and pubic

Figure 2. Plasma gonadotropin patterns and bioactive FSH (B-FSH) in response to pulsatile administration of synthetic GnRH in case 2. Arrows denote administration of synthetic GnRH pulses. B-FSH was measured before and after these GnRH pulses (top panel). B-FSH values are expressed as $\mathrm{ng} / \mathrm{mL}$ of the $\mathrm{hFSH} 3$ standard. Immunoreactive LH and FSH are presented in the bottom panel.

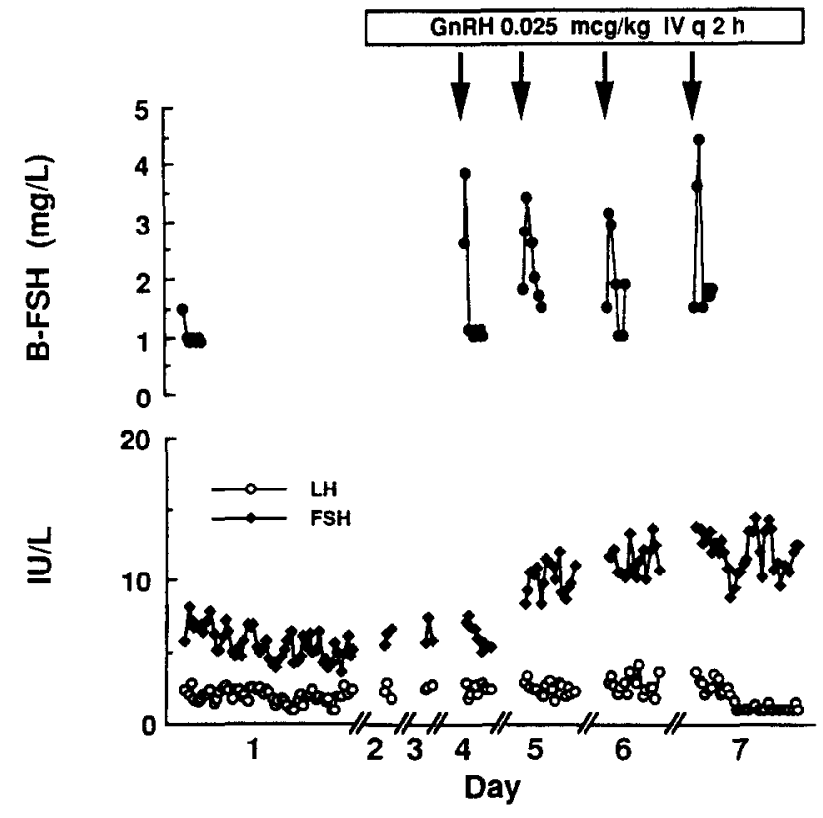

hair. Testes were small, measuring $3 \mathrm{~cm}$ on the longer axis, and the scrotum was poorly developed.

\section{- Case Presentation 3}

An infant boy presented at 2 weeks of age with frequent vomiting, weight loss, dehydration, and apathy. His case history was presented before (Virdis et al. 1983) and only a brief summary is outlined here. His brother died suddenly at the age of 2 months; an autopsy revealed interstitial pneumonia, but the adrenal glands were not evaluated. Birth weight of this infant (case 3) was $4300 \mathrm{~g}$. Laboratory evaluation revealed hyperkalemia and hyponatremia. Primary adrenal insufficiency was diagnosed, based on low concentrations of urinary 17 hydroxysteroids and 17-ketosteroids, and plasma aldosterone, cortisol, and 17hydroxyprogesterone, as well as unresponsiveness to ACTH stimulation. Therapy was started with hydrocortisone succinate and a mineralocorticoid (fludrocortisone). He grew along the third percentile until 12 years of age, when his relative growth slowed further. Skeletal maturation had been $4^{1 / 2}$ years at $5^{8 / 12}$ years of age, and the delay in skeletal maturation increased progressively; at $12^{1 / 2}$ years of age, his bone age was $9^{1 / 2}$ years. Serum androgen concentrations [dehydroepiandrosterone (DHEA), DHEA sulfate, androstenedione, and testosterone] were measured periodically beginning at 12 years of age and were low. At $14 \frac{5}{12}$ years, slight development of pubic hair growth was noted, but no further pubertal changes developed. Between $141 / 2$ and 16 years of age, he had a slight increase in serum testosterone, but serum testosterone fell to within the prepubertal range by age 17 years. Results of a synthetic GnRH stimulation test (2.5 $\mu \mathrm{g} / \mathrm{kg}$ i.v.) suggested gonadotropin deficiency, as LH and FSH concentrations did not increase. He was admitted to the Clinical Research Center for a 5-day GnRH stimulation protocol during which intravenous bolus injections of $\mathrm{GnRH}$ $(0.025 \mu \mathrm{g} / \mathrm{kg}$ per dose) were given every 2 h. His plasma gonadotropin responses are depicted in Figure 3. Plasma immunoreactive and bioactive FSH concentrations increased, but LH concentrations as well as LH $\alpha$ subunit (data not shown) remained low. At 18 years of age, treatment with a depot preparation of 


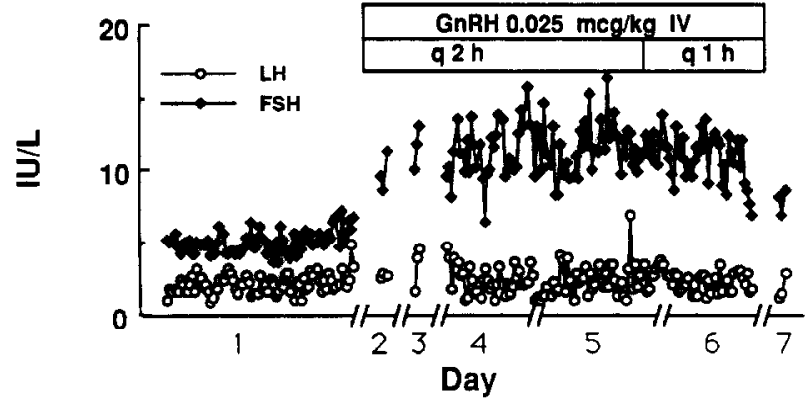

Figure 3. Plasma immunoreactive gonadotropin patterns in response to pulsatile synthetic GnRH administration in case 3.

testosterone esters was begun, and secondary sex characteristics developed over the ensuing months.

\section{- Discussion}

Idiopathic congenital adrenal hypoplasia is a rare disorder with an overall estimated frequency of $1: 12,500$ live births (Kelch et al. 1984). Two distinct forms have been identified, based on the histologic appearance of the adrenal glands. The miniature adult form, also called anencephalic or secondary form, is characterized by small adrenal glands with normal architecture. There is a well-defined permanent zone and a greatly reduced fetal zone. This form is believed to be secondary to ACTH deficiency, such as seen in anencephalic infants, infants of mothers who received therapy with glucocorticoids during pregnancy, or infants with congenital anomalies of the hypothalamus or pituitary gland. This secondary form is usually sporadic, although few cases of autosomal recessive inheritance have been reported (Kelch et al. 1984; Prader et al. 1975); the observed association of achalasia and adrenal hypoplasia in a subset of familial cases suggests that autosomal recessive congenital adrenal hypoplasia may be heterogeneous (Allgrove et al. 1978).

The primary, or cytomegalic, form is characterized by an absence of the permanent zone of the adrenal cortex; the cytomegalic cells are larger than normal fetal adrenal cells and have nuclear inclusions due to cytoplasmic invaginations. The primary form of congenital adrenal hypoplasia is an X-linked disorder usually manifesting as adrenal insufficiency early in infancy, with low serum concentrations of glucocorticoids, mineralocorticoids, and androgens. Failure to respond to ACTH stimulation is characteristic. As illustrated by all three case presentations, hypogonadotropic hypogonadism is noted at the expected time of pubertal maturation (Prader et al. 1975; Virdis et al. 1983; Hay et al. 1981; Kelly et al. 1977; Golden et al. 1977; Lippe and Golden 1978; Petersen et al. 1982).

Clinical signs and symptoms of infants with adrenal hypoplasia include poor feeding, failure to gain weight, hyperpigmentation, vomiting, diarrhea, vascular collapse, and sudden death. Dehydration, hyponatremia, hyperkalemia, acidosis, and hypoglycemia are common biochemical findings characteristic of combined gluco- and mineralocorticoid deficiency. These signs and symptoms are indistinguishable from those seen in male infants with the salt-wasting form of congenital adrenal hyperplasia due to 21hydroxylase deficiency. A clue for differentiation between these two possible diagnoses in a male infant is the size of the genitalia. In infants with 21-hydroxylase deficiency, the testicles are normal in size and the phallus may be larger than normal owing to elevated plasma androgen concentrations. In contrast, the testes and phallus are usually small in infants who have adrenal hypoplasia (Kelch et al. 1984). With prompt diagnosis and replacement therapy, survival has increased greatly (Kelch et al. 1984; Virdis et al. 1983). Although the majority of patients present in infancy, some patients similar to case 2 do not present with classic signs and symptoms of adrenal insufficiency until the later childhood years (Hay et al. 1981, case 2; Kelly et al. 1977; Golden et al. 1977).

In a male infant, a presumptive diagnosis of congenital adrenal hypoplasia may be reached by determination of serum concentrations of cortisol, aldosterone, 17-hydroxyprogesterone, 17hydroxypregnenolone, and DHEA and its sulfate, all of which are low. Plasma ACTH concentrations are increased. Demonstration of failure to increase serum concentrations of the abovementioned steroids following administration of ACTH strongly supports the diagnosis (Kelch et al. 1984; Kelly et al. 1977; Petersen et al. 1982). Distinguishing patients with congenital adrenal hypoplasia from those with hereditary ACTH unresponsiveness, although sometimes difficult, is usually made clinically, as the latter patients do not have significant mineralocorticoid deficiency nor are they gonadotropin deficient (Kelch et al. 1972).

Replacement therapy with hydrocortisone succinate, fludrocortisone, and salt $(\mathrm{NaCl})$ should be initiated promptly and monitored carefully (Kelch et al. 1984; Hughes 1988). Careful attention to growth velocity and rate of skeletal maturation is essential since, unlike the 21-hydroxylase-deficiency form of congenital adrenal hyperplasia, there is no useful biochemical marker for adequacy of treatment of patients who have congenital adrenal hypoplasia.

As gonadotropin deficiency is commonly associated with the primary form of adrenal hypoplasia, the patients must be followed closely for physical signs of pubertal maturation (Kelch et al. 1984; Petersen et al. 1982; Zachman et al. 1980). When a patient with primary adrenal hypoplasia shows no signs of sexual maturation by 13-14 years of age, hypogonadotropic hypogonadism should be suspected. Low serum concentrations of $\mathrm{LH}, \mathrm{FSH}$, and testosterone are usually found, and the responses of serum gonadotropin concentrations to a single GnRH bolus are usually low (Dunkel et al. 1985). A stimulation test with hCG, performed by administration of hCG $2000 \mathrm{U}$ i.m. twice a week for 4-6 weeks, and determination of serum testosterone concentrations before and after the hCG therapy are recommended to evaluate testicular function before therapy with androgens is started (Dunkel et al. 1985). The majority of reported patients had normal testicular responses to hCG (Kelch et al. 1984; Virdis et al. 1983, case 1; Hay et al. 1981; Kelly et al. 1977; Golden et al. 1977; Lippe and Golden 1978; Petersen et al. 1982; Zachman et al. 1980; Gordon et al. 1984; Kikuchi et 
al. 1987; Bovet et al. 1988; Kruse et al. 1984; Partsch and Sippell 1989), though some patients had suboptimal responses (case 3; Virdis et al. 1983, case 2; Hay et al. 1981, case 1). We prefer to use a long-acting testosterone ester rather than hCG to achieve masculinization because of lower cost and lower frequency of injections. Initially, we start with 50-75 mg of testosterone enanthate every 4 weeks, and gradually increase the dose and frequency to $200 \mathrm{mg}$ every 3 weeks over a 2- to 3-year period.

In an attempt to explain the endocrinologic basis of this disorder, Golden and colleagues (1977) proposed that a prenatal delay in the development of hypothalamic function might lead to the absence of ACTH during the critical period of adrenal development and also to permanent gonadotropin deficiency. There is little evidence to support this theory, however, and it seems unlikely that ACTH deficiency is involved in the pathogenesis as ACTH secretion is normal or increased in patients with primary adrenal hypoplasia. Also, this theory does not explain the finding of patients who have a delayed onset such as in case 3 and others (Hay et al. 1981; Kelly et al. 1977) who, based on clinical data, were presumed to have had normal function of the adrenal gland until the childhood years. Moreover, normal adrenal function was documented by determination of normal urinary excretion of adrenocorticosteroids in a patient who had a positive family history and who developed adrenal insufficiency at the age of 10 years (Golden et al. 1977; Lippe and Golden 1978). Similarly, Sills and colleagues (1983) reported a case of a male infant who demonstrated a progressive decline in adrenal function: at 2 months of age, he had a normal cortisol response to ACTH, but his adrenal function declined and, by 3 years of age, he had no response to ACTH.

Whether the hypogonadotropic hypogonadism in patients who have congenital adrenal hypoplasia is the result of pituitary or hypothalamic dysfunction has not been settled. Initial reports of the association of congenital adrenal hypoplasia with isolated gonadotropin deficiency documented poor gonadotropin responses to a single dose of synthetic $\mathrm{GnRH}$ in the majority of the patients (Kelch et al. 1984; Prader et al. 1975; Virdis et al. 1983; Hay et al. 1981; Kelly et al. 1977; Golden et al. 1977), but not all (Petersen et al. 1982). This did not differentiate primary pituitary from hypothalamic dysfunction, as most patients who have isolated gonadotropin deficiency, due to GnRH deficiency, as well as patients who have primary pituitary dysfunction, respond poorly or not at all to a single dose of synthetic GnRH (Dunkel et al. 1985).

To try to differentiate between hypothalamic and pituitary gland dysfunction, more prolonged administration of $\mathrm{GnRH}$ is required (Kelch et al. 1984; Barkan et al. 1985). Several recent reports have suggested pituitary gland dysfunction as the primary defect, based upon lack of gonadotropin responses to prolonged, pulsatile stimulation with synthetic GnRH (Gordon et al. 1984; Kikuchi et al. 1987; Bovet et al. 1988). Gordon and associates (1984) described striking differences in responses to GnRH between a patient with Kallman's syn-

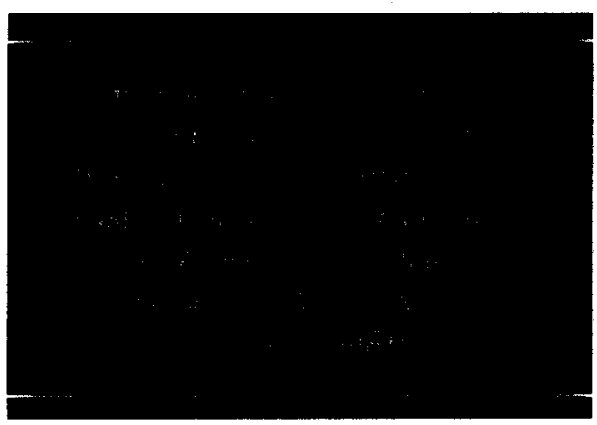

drome, whose LH and FSH rose to within the normal range after 1 week of therapy, and a patient with congenital adrenal hypoplasia in whom only minimal increases of LH and FSH were found after 16 weeks of therapy. Serum testosterone concentrations increased in the patient with Kallman's syndrome, but not in the patient with adrenal hypoplasia. Similar observations of failure to produce pubertal LH and FSH secretion in patients with X-linked adrenal hypoplasia after prolonged treatment with pulsatile GnRH have been reported by others (Kikuchi et al. 1987; Bovet et al. 1988). The possibility of primary pituitary dysfunction is further supported by the finding of Marsden and Zakkhour (1978), who reported cytomegalic changes in the pituitary gland of an infant who dicd of congenital adrenal hypoplasia.

In contrast, Partsch and Sippell (1989) reported successful induction of pubertal concentrations of LH, FSH, and testosterone 2-3 months after initiation of therapy with pulsatile synthetic $\mathrm{GnRH}$, suggesting hypothalamic dysfunction as the principal defect in their patient with congenital X-linked adrenal hypoplasia. At least initially, patients with severe endogenous GnRH deficiency respond to exogenous GnRH with the preferential secretion of FSH (Barkan et al. 1985). This may be important, as the patients studied by Kikuchi et al. (1987) had increased serum FSH concentrations and normalization of FSH responses to synthetic GnRH although serum LH hyporesponsiveness persisted. Thus, the increases in serum FSH noted in our cases (Figures 1-3), as well as others, support the possibility of a primary hypothalamic abnormality (Kruse et al. 1984). Finally, in case 2 , serum bioactive FSH responses to synthetic GnRH increased after pulsatile GnRH therapy similar to findings in patients with idiopathic hypogonadotropic hypogonadism (Padmanabhan et al. 1988).

Differences in gonadotropin responses of patients with congenital adrenal hypoplasia to the prolonged administration of synthetic GnRH might be attributed to differences in the treatment protocols, but this seems unlikely, since the frequency of pulsatile injections varied only between 90 and $120 \mathrm{~min}$ and the dosage range was $0.05-0.2 \mu \mathrm{g} / \mathrm{kg}$ per dose (Bovet et al. 1988; Gordon et al. 1984; Kikuchi et al. 1987; Kruse et al. 1984; Partsch and Sippell 1989). Alternatively, since histologic or precise genetic confirmation of the diagnosis has been unavailable in patients who have received pulsatile GnRH, the syndrome of congenital adrenal hypoplasia with isolated gonadotropin deficiency may be genetically heterogeneous. Thus far, no more than one affected individual from an involved family has received prolonged stimulation with synthetic GnRH. It will be important to determine whether GnRH responsiveness differs among af fected boys within a kindred and whether different families have different genetic abnormalities.

Appropriate genetic counseling is an important component of the overall management of families with an affected child. To assist in differentiating between the autosomal recessive and the $\mathrm{X}$-linked forms of congenital adrenal hypoplasia, a detailed pedigree of the proband's family should be obtained, 
with particular attention to the previous occurrence of neonatal or unexplained deaths, similarly affected individuals within the maternal lineage, and the possibility of consanguinity. Since adrenal insufficiency can precede neurologic symptoms by months to years in individuals with X-linked adrenoleukodystrophy (ALD), a distinctly different $\mathrm{X}$-linked disease mapped to Xq28 (Mandel et al. 1989), pertinent laboratory assays, such as serum long-chain fatty-acid quantification (elevated in ALD), may be indicated to assist in making an accurate diagnosis. In families with a documented affected male infant with X-linked adrenal hypoplasia, the recurrence risk for a subsequent male infant may be as high as $50 \%$, with a similar risk for a female sibling of being a gene carrier.

Hammond et al. (1985) suggested that the locus for X-linked congenital adrenal hypoplasia and that for glycerol kinase were located on the short arm of the $\mathrm{X}$ chromosome within the region $\mathrm{Xp11.2-}$ p21, based on the findings of an interstitial Xp deletion with breakpoints at $\mathrm{Xp} 11.2$ and $\mathrm{Xp21}$ in a phenotypically normal mother of a male infant who died at $36 \mathrm{~h}$ of cytomegalic adrenal hypoplasia with glyceroluria (indicating glycerol kinase deficiency) and ornithine transcarbamoyltransferase deficiency. The molecular genetic analyses of multiple unrelated affected males with observed cytologic and submicroscopic interstitial deletions have localized the X-linked congenital adrenal hypoplasia locus to region Xp21.3-p21.2 (Bartley et al. 1986; Francke et al. 1987; Mandel et al. 1989) between two anonymous DNA sequences, DXS67 and DXS268, with a gene order of Xpter-DSX67-AH-GK-DXS268-DMDOTC-Xcen (Xp telomere, Xpter; adrenal hypoplasia, AH; glycerol kinase, GK; Duchenne muscular dystrophy, DMD; ornithine transcarbamoyltransferase, OTC; and X centromere, Xcen) (Goonewardena et al. 1989). These studies indicate that it may be feasible to use polymorphic DNA markers closely linked to the adrenal hypoplasia locus to provide prenatal diagnostic studies to families with a previously affected member.

Not infrequently, patients with $\mathrm{X}$ linked adrenal hypoplasia have been found to be affected with additional $\mathrm{X}$-linked diseases such as Duchenne muscular dystrophy, glycerol kinase de- ficiency, ornithine transcarbamoyltransferase deficiency, and mental retardation as a consequence of having an interstitial deletion within Xp21 disrupting a number of neighboring, or contiguous, genetic loci (Hammond et al. 1985; Bartley et al. 1986; Francke et al. 1987). These patients present with a variety of clinical problems depending on which contiguous genes have been disrupted; the various clinical syndromes resulting from different overlapping deletions have been referred to as contiguous deletion syndromes (Schmickel 1986; Emanuel 1988). Because X-linked adrenal hypoplasia is not uncommonly a consequence of an $\mathrm{X}$-chromosome deletion, affected patients should be evaluated for clinical signs of other diseases mapped to Xp21 and, when appropriate, diagnostic studies, including a karyotype and urine organic acids, should be obtained.

In a male infant from an affected kindred, the finding of normal results of adrenal function at birth does not rule out the possibility of X-linked adrenal hypoplasia, as onset of symptoms may be delayed in some patients (case 1; Hay et al. 1981; Kelly et al. 1977; Golden et al. 1977; Sills et al. 1983), and thus careful follow-up is indicated.

References

Allgrove J, Clayden GS, Grant DB, Macauley JC: 1978. Familial glucocorticoid deficiency with achalasia of the cardia and deficient tear production. Lancet 1:1284-1286.

Barkan AL, Reame NE, Kelch RP, Marshall JC: 1985 . Idiopathic hypogonadotropic hypogonadism in men: dependence of the hormone responses to gonadotropin-releasing hormone on the magnitude of the endogenous GnRH secretory defect. J Clin Endocrinol Metab 61:1118-1125.

Bartley JA, Patil S, Davenport S, Goldstein D, Pickens J:1986. Duchenne muscular dystrophy, glycerol kinase deficiency, adrenal insufficiency associated with $\mathrm{Xp} 21$ interstitial deletion. J Pediatr 108:189-192.

Bovet P, Reymond MI, Gomez F: 1988. Lack of gonadotropic response to pulsatile gonadotropin-releasing hormone in hypogonadotropic hypogonadism associated to congenital adrenal hypoplasia. J Endocrinol Invest 11:201-204.

Dunkel L, Perheentupa J, Virtanen M, Maenpaa J: 1985. GnRH and hCG tests are both necessary in differential diagnosis of male delayed puberty. Am J Dis Child 139:494-498.

Emanuel BS: 1988. Molecular cytogenetics: toward dissection of the contiguous gene syndromes. Am J Hum Genet 43:575-578.

Francke U, Harper JF, Darras BT, et al.: 1987. Congenital adrenal hypoplasia, myopathy, glycerol kinase deficiency: molecular genetic evidence for deletions. Am J Hum Genet 40:212-228.

Golden MP, Lippe BM, Kaplan SA: 1977. Congenital adrenal hypoplasia and hypogonadotropic hypogonadism. Am J Dis Child 131:1117-1118.

Goonewardena P, Dahl N, Ritzen M, Van Ommen GJB, Pettersson U: 1989. Molecular Xp deletion in a male: suggestion of a locus for hypogonadotropic hypogonadism distal to the glycerol kinase and adrenal hypoplasia loci. Clin Genet 35:5-12.

Gordon D, Cohen HN, Beastall GH, Hay ID, Thomson JA: 1984. Contrasting effects of subcutaneous pulsatile gonadotropin-releasing hormone therapy in congenital adrenal hypoplasia and Kallman's syndrome. Clin Endocrinol 21:597-603.

Hammond J, Howard NJ, Brookwell R, PurvisSmith S, Wilcken B, Hoogenraad N: 1985. Proposed assignment of loci for X-linked adrenal hypoplasia and glycerol kinase genes. Lancet 1:54.

Hay ID, Smail PJ, Forsyth CC: 1981. Familial cytomegalic adrenocortical hypoplasia: an X-linked syndrome of pubertal failure. Arch Dis Child 56:715-721.

Hughes IA: 1988. Management of congenital adrenal hyperplasia. Arch Dis Child 63:13991404.

Kelch RP, Kaplan SL, Biglieri EG, Daniels GH, Epstein CG, Grumbach MM: 1972. Hereditary adrenal cortical unresponsiveness to ACTH. J Pediatr 81:726-736.

Kelch RP, Virdis R, Rapaport R, Greig F, Levine LS, New MI: 1984. Congenital adrenal hypoplasia. Pediatr Adolesc Endocrinol 13:156-161.

Kelly WF, Joplin GF, Pearson GW: 1977. Gonadotropin deficiency and adrenocortical insufficiency in children: a new syndrome. Br Med J 2:98.

Kikuchi K, Kaji M, Mikawa H, Shigematsu Y, Sudo M: 1987 . Failure to induce puberty in a man with $\mathrm{X}$-linked congenital adrenal hypoplasia and hypogonadotropic hypogonadism by pulsatile administration of lowdose gonadotropin-releasing hormone. Acta Endocrinol (Copenh) 114:153-160.

Kruse K, Sippell WG, Schnakenburg KV: 1984. Hypogonadism in congenital adrenal hypoplasia: evidence for a hypothalamic origin. J Clin Endocrinol Metab 58:12-17.

Lippe BM, Golden MP: 1978. Pubertal failure in congenital adrenal hypoplasia. Lancet 1:392-393.

Mandel J-L, Willard HF, Nussbaum RL, Romeo G, Puck JM, Davies KE: 1989. Report of the 
committee on the genetic constitution of the $\mathrm{X}$ chromosome. Cytogenet Cell Genet 51:384-437.

Marsden HB, Zakkhour HD: 1978. Cytomegalic adrenal hypoplasia with pituitary cytomegaly. Virchows Arch [A] 378:105-110.

Padmanbhan V, Kelch RP, Sonstein J, Foster CM, Beitins IZ: 1988. Bioactive FSH responses to intravenous $\mathrm{GnRH}$ in boys with idiopathichypogonadotropichypogonadism. J Clin Endocrinol Metab 67:793-800.

Partsch CJ, Sippell WG: 1989. Hypothalamic hypogonadism in congenital adrenal hy- poplasia. Horm Metabol Res 21:623-625.

Petersen KE, Bille T, Jacobsen BB, Iversen T: 1982. X-linked congenital adrenal hypoplasia: a study of five generations of a Greenlandic family. Acta Paediatr Scand 7:947-951.

Prader A, Zachman M, Illig R: 1975. Luteinizing hormone deficiency in hereditary congenital adrenal hypoplasia. J Pediatr $86: 421-422$.

Schmickel RD: 1986. Contiguous gene syndromes: a component of recognizable syndromes. J Pediatr 109:231-241.
Sills IN, Voorhess ML, MacGillivray $\mathrm{MH}$, Peterson RE: 1983. Prolonged survival without therapy in congenital adrenal hypoplasia. Am J Dis Child 137:1186-1188.

Virdis R, Levine LS, Levy D, Pang S, Rapaport R, New MI: 1983. Congenital adrenal hypoplasia: two new cases. J Endocrinol Invest 6:51-54.

Zachman M, Illig R, Prader A: 1980. Gonadotropin deficiency and cryptorchidism in three prepubertal brothers with congenital adrenal hypoplasia. J Pediatr 97:255-257.

TEM

\section{Why wait for it to circulate? Read your own copy of}

\section{Trends in Endocrinology and Metabolism!}

If you share TEM with others at work, you know that what goes around doesn't always come around. And if it does, articles are often dated or sometimes even clipped.

Why miss a single issue, or wait weeks to read one? By starting your personal subscription, for just $\$ 48$ you'll be guaranteed to read each and every issue.

Trends in Endocrinology and Metabolism's short reviews on "hot" topics can make a difference in your practice and research. Commissioned clinicians and researchers report on advances in reproductive endocrinology, developmental endocrinology, metabolism, biochemistry, diabetes and insulin action, thyroid hormone action, obesity, neuroendocrinology, molecular and cellular biology and host of other areas.

From resident to experienced clinician, post-doc to lab director, graduate student to professor -all can rely on TEM for the latest updates and insights into the cutting edge of endocrinology.

USE THE BUSINESS REPLY CARDS IN THIS ISSUE TO ORDER YOUR PERSONAL SUBSCRIPTION TODAY-.OR CALL ELSEVIER CUSTOMER SERVICE AT (212) 633-3950, FAX (212) 633-3880. 